\title{
On Simple Singular N-Flat Modules
}

Raida D. Mahmood

raida.1961@uomosul.edu.iq

College of Computer Sciences and Mathematics

University of Mosul, Mosul, Iraq
Dina A. Hadid

College of Basic Education

University of Duhok, Duhok, Iraq

Received on: 04/04/2011

Accepted on: 16/05/2011

\section{ABSTRACT}

Let $\mathrm{I}$ be a right ideal of a ring $\mathrm{R}$, then $\mathrm{R} / \mathrm{I}$ is right $\mathrm{N}$-flat module if and only if for each $a \in I$, there exists $b \in I$ and a positive integer $\mathrm{n}$ such that $a^{n} \neq 0$ and $a^{n}=b a^{n}$.In this paper, we first introduce and characterize rings whose every simple singular right $\mathrm{R}$-module is $\mathrm{N}$ - flat. Next, we investigate the strong regularity of rings whose every simple singular right $\mathrm{R}$ - module is $\mathrm{N}$-flat. It is proved that :

$\mathrm{R}$ is strongly regular ring if and only if $\mathrm{R}$ is a wjc , MERT and 2 - primal ring whose simple singular right $\mathrm{R}$ - module is $\mathrm{N}$ - flat.

Let $\mathrm{R}$ be a wjc ring satisfying condition (*). If every simple singular right $\mathrm{R}$ module is $\mathrm{N}$-flat .Then, the Center of $\mathrm{R}$ is a regular ring.

Keywords: N-Flat, MC2 - ring, WPSI ring , GQ - injective , CAM - ring

$$
\begin{aligned}
& \text { حول المقاسات البسيطة المنفردة المسطحة من النمط N } \\
& \text { دينا امير حديد } \\
& \text { كلية التربية الاساسية } \\
& \text { رائدة داؤد محمود } \\
& \text { جامعة دهوك، دهوك، العراق } \\
& \text { كلية علوم الحاسوب والرياضيات }
\end{aligned}
$$

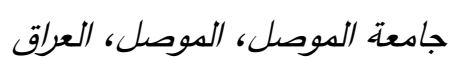

$$
\begin{aligned}
& \text { تاريخ قبول البحث: 2011/05/16 } \\
& \text { تاريخ استلام البحث: 2011/04/04 }
\end{aligned}
$$

\section{الملخص}

ليكن ا مثالي أيمن في الحلقة R ، فإن R/l يكون مقاساً مسطحاً من النمط - N أيمن إذا وفقط إذا لكل

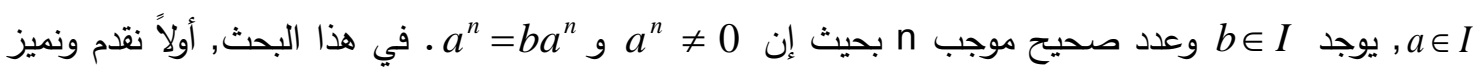
الحلقات التي فيها كل مقاس منفرد بسيط مسطحاً من النمط - N ـ ونقوم بالبحث عن الحلقات المنتظمة بقوة والتي

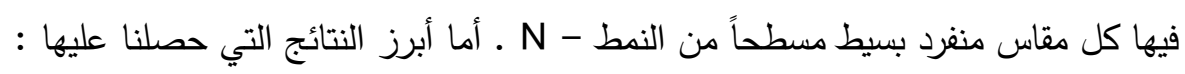

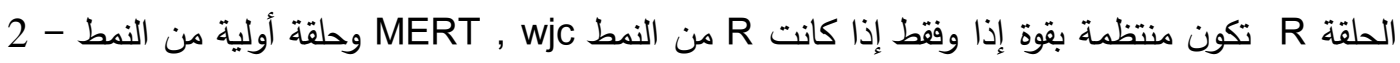

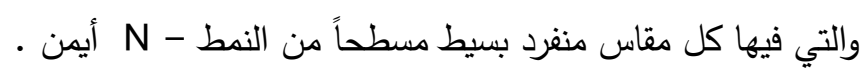

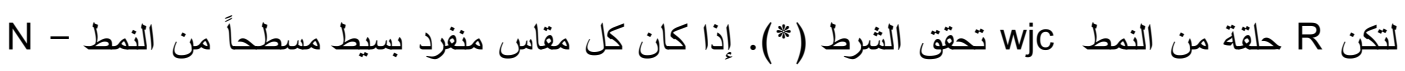
أيمن. فإن مركز الحلقة R يكون منتظماً. الكلمات المفتاحية: مسطحة من النمط-N، حلقة من النمط-MC2، حلقة WPSI، الغامرة من النمط-GQ، حلقة 


\section{Introduction}

Throughout this paper, $\mathrm{R}$ denotes an associative ring with identity, and all modules are unitary right $\mathrm{R}$ - module. For any nonempty subset $\mathrm{X}$ of a ring $\mathrm{R}, r(X)$ and $l(X)$ denote the right annihilator of $\mathrm{X}$ and the left annihilator of $\mathrm{X}$, respectively . If $X=\{a\}$, we use the abbreviation $l(a)$ and $r(a)$. We write $J(R), Z(R)$ and $Y(R)$ for the Jacobson radical of $\mathrm{R}$, the left singular ideal of $\mathrm{R}$ and the right singular ideal of $\mathrm{R}$, respectively.

Recall that, Let $\mathrm{I}$ be a right ideal of a ring $\mathrm{R}$, then $\mathrm{R} / \mathrm{I}$ is a flat right $\mathrm{R}$ - module if and only if for each $a \in I$, there exists $b \in I$ such that $a=b a$ ( cf. [7] , [8],[3] ). The generalization of flat module to $\mathrm{N}$-flat module is performed as follows : Let I be a right ideal of a ring $\mathrm{R}$, then $\mathrm{R} / \mathrm{I}$ is a right $\mathrm{N}$-flat module if and only if for each $a \in I$, there exists $b \in I$ and a positive integer $\mathrm{n}$ such that $a^{n} \neq 0$ and $a^{n}=b a^{n}$ [1]. And, in [1], we give a lot of characterizations of right $\mathrm{N}$ - flat . For example, $J(R) \cap Y(R)=(0)$, if $\mathrm{R}$ satisfying condition $(*)$ whose every simple singular right $\mathrm{R}$-module is $\mathrm{N}$-flat .

The ring $R$ is said to be 2 - primal if $N(R)=P(R)$, where $N(R)$ is the set of all nilpotent element and $P(R)$ is the prime radical of $R$ [4] . The ring $R$ is called right wjc ring, if $a R b=0$ for $a, b \in R$, implies $b R a=0$ [12]. The ring $\mathrm{R}$ is said to be reduced if $\mathrm{R}$ has no nonzero nilpotent element. The ring $\mathrm{R}$ is called right SXM [12], if for each $0 \neq a \in R, r(a)=r\left(a^{n}\right)$ for all a positive integer $\mathrm{n}$ satisfying $a^{n} \neq 0$. For example, reduced rings are right SXM rings. The concept of regular rings was introduced in (1936) by Von Neumann [9] . The ring R is called MERT , if every essential maximal right ideal of $\mathrm{R}$ is an ideal. The ring $\mathrm{R}$ is called regular (strongly regular) ring, if for every $a \in R$, there exists $b \in R$ such that $a=a b a\left(a=a^{2} b\right)$.The ring $\mathrm{R}$ is called right weakly regular ring, if for every $a \in R, a \in a R a R$ [7] .

\section{Characterizations of Simple Singular $\mathbf{R}$ - Module is $\mathbf{N}$ - Flat}

This section is devoted to study rings whose every simple singular right R-module is $\mathrm{N}$-flat with some of their basic properties . On the other hand,we characterized MC2 rings terms of simple singular right $\mathrm{R}$ - modules is $\mathrm{N}$ - flat .

\section{Definition 2.1 [10]}

The ring $\mathrm{R}$ is called right $\mathrm{MC} 2$ ring. If $e R a=0$ implies $a R e=0$, where $a \in R$ , $e^{2}=e \in R$ and $K \cong e R$ is minimal right ideal of $\mathrm{R}$, or equivalently if $e R$ are minimal, $e^{2}=e \in R$; then $K=g R$ for some $g^{2}=g \in R$.

We consider the condition (*); R satisfies $l(a) \subseteq r(a)$ for every $a \in R$.

\section{Theorem 2.2}

Let $\mathrm{R}$ be a right $\mathrm{MC} 2$ ring satisfying condition (*). If every simple singular right $\mathrm{R}$ - module is $\mathrm{N}$ - flat, then $Z(R)=(0)$.

Proof : Suppose that $Z(R) \neq(0)$, then there exists $0 \neq a \in Z(R)$ such that $a^{2}=0$.We claim that $Z(R)+r(a)=R$. Otherwise, there exists a maximal right ideal $\mathrm{M}$ such that $Z(R)+r(a) \subseteq M$. If $\mathrm{M}$ is not essential, then $M=r(e), e^{2}=e \in R$. Hence $e a=0$ because $a \in r(a) \subseteq M=r(e)$. If $e R a \neq 0$, then $e R a R=e R$ because $e R$ is a minimal right ideal of $\mathrm{R}$. Since $a \in Z(R), \quad e R a R \subseteq Z(R)$, then $e \in Z(R)$, which is a contradiction. Hence, $a R e=0$.Since $\mathrm{R}$ right $\mathrm{MC} 2$ ring, then $a R e=0$, 
$e \in r(a) \subseteq M \subseteq r(e)$, which is a contradiction . Hence, $\mathrm{M}$ is essential in $\mathrm{R}$. Thus, $\mathrm{R} / \mathrm{M}$ is $\mathrm{N}$ - flat, then there exists a positive integer $\mathrm{n}$ and $b \in M$ such that $a^{n} \neq 0$ and $a^{n}=b a^{n}$. Since $a^{2}=0$, then $n=1$ and therefore $(1-b) \in r(a) \subseteq M$ and $1 \in M$, which is a contradiction. Hence, $Z(R)+r(a)=R$. Write $1=x+y$, where $x \in Z(R)$ and $y \in r(a)$, then $a=a x$. Since $x \in Z(R)$ and $l(x) \cap l(1-x)=0, l(1-x)=0$. Thus, $a=0$ because $a \in l(1-x)$, which is a contradiction. This implies that $Z(R)=(0)$

\section{Theorem 2.3}

Let $\mathrm{R}$ be a ring whose every simple singular right $\mathrm{R}$-module is $\mathrm{N}$ - flat, satisfying condition $(*)$.Then,$Z(R) \cap Y(R)=(0)$.

Proof : Suppose that $Z(R) \cap Y(R) \neq(0)$, then there exists $0 \neq a \in Z(R) \cap Y(R)$ such that $a^{2}=0$. We claim that $Z(R)+r(a)=R$. Otherwise, there exists a maximal essential right ideal $\mathrm{M}$ such that $Z(R)+r(a) \subseteq M$. Thus, $\mathrm{R} / \mathrm{M}$ is $\mathrm{N}$ - flat ,then there exists $b \in M$ and a positive integer $\mathrm{n}$ such that $a^{n} \neq 0$ and $a^{n}=b a^{n}$. Since $a^{2}=0$, then $n=1$, and therefore $a=b a$, which implies that $(1-b) \in l(a) \subseteq r(a) \subseteq M, 1 \in M$, which is a contradiction. Hence, $Z(R)+r(a)=R$. Write $1=x+y, x \in Z(R)$ .Thus, $l(1-x)=0, l(x) \cap l(1-x)=0$ and $x \in Z(R)$. Since $a=a x$, then $y \in r(a)$ This implies that contradiction. a, which is $a \in l(1-x)$ because $a=0$ 口. $Z(R) \cap Y(R)=(0)$

\section{Lemma 2.4 [1]}

If $\mathrm{R}$ is a ring satisfying condition (*) whose every simple singular right $\mathrm{R}$ - module is $\mathrm{N}$ - flat, then $J(R) \cap Y(R)=(0)$

According to [11] , a right $\mathrm{R}$ - module $\mathrm{M}$ is said to be right weakly principally small injective (WPSI) if for any $0 \neq a \in J(R)$, there exists a positive integer $\mathrm{n}$ such that $a^{n} \neq 0$ and any right $\mathrm{R}$ - homomorphism from $a^{n} R \rightarrow M$ can be extended to $R \rightarrow M$. A ring $\mathrm{R}$ is called right WPSI if $R_{R}$ is a right WPSI.

\section{Definition 2.5 [6]}

The ring $\mathrm{R}$ is said to be right(left) mininjective if every $\mathrm{R}$ - homomorphism from a minimal right(left) ideal of $\mathrm{R}$ to $R_{R}$ can be extended from $\mathrm{R}$ to $R_{R}$.

\section{Lemma 2.6 [11]}

If $\mathrm{R}$ is a left (right) WPSI ring, then :

1. $J(R) \subseteq Z(R)(J(R) \subseteq Y(R))$.

2. $\mathrm{R}$ is a left (right) mininjective ring

\section{Corollary 2.7}

Let $\mathrm{R}$ be a right MC2 ring, left WPSI satisfying condition (*). If every simple singular $\mathrm{R}$ - module is $\mathrm{N}$ - flat, then $J(R)=(0)$.

Proof : From Lemma 2.6, we have $J(R) \subseteq Z(R)$. So $J(R)=(0)$ because $Z(R)=(0)$ , by Theorem 2.2

\section{Proposition 2.8}

Let $\mathrm{R}$ be a right WPSI ring, whose every simple singular right R-module is $\mathrm{N}$ flat and satisfying condition $(*)$. Then, $Z(R)=(0)$. 
Proof : By Lemma $2.4, J(R) \cap Y(R)=(0)$. Since $\mathrm{R}$ is a right WPSI ring then , $J(R) \subseteq Y(R)$ Lemma 2.6, so $J(R)=J(R) \cap Y(R)=(0)$ and $\mathrm{R}$ is a right mininjective ring Lemma 2.6.Therefore, $\mathrm{R}$ is a right MC2 ring [ 5, Theorem 1.14 ]. Hence , by Theorem $2.2 . Z(R)=(0)$

\section{Certain Rings Whose every Simple Singular $\mathbf{R}$ - Module is $\mathbf{N}$ - Flat}

In this section, we study the relation between rings whose every simple singular right $\mathrm{R}$ - modules are $\mathrm{N}$ - flat, reduced rings and strongly rings by adding some types of rings such as wjc and $\mathrm{MC} 2$ rings, and other types of rings .

\section{Theorem 3.1}

Let $\mathrm{R}$ be a wjc ring satisfying condition (*). If every simple singular right $\mathrm{R}$ - module is $\mathrm{N}$ - flat. Then, $\mathrm{R}$ is a semiprime .

Proof : Suppose that $\mathrm{I}$ is an ideal of $\mathrm{R}$ with $I^{2}=0$. If $I \neq 0$, then there exists $0 \neq a \in I$ such that $a I=0$ and so $a^{2}=0$. First observe $\mathrm{r}(\mathrm{a})$ which is an essential right ideal of $\mathrm{R}$. if not, then there exists a nonzero right ideal $\mathrm{K}$ of $\mathrm{R}$ such that $r(a) \cap K=0$. Since $a K I \subseteq a I=0, K I \subseteq r(a) \cap K=0$. Since $\mathrm{R}$ is a wjc ring, $I K=0$ . Hence, $a K=0$ and so $K \subseteq r(a) \cap K=0$, which is a contradiction .Hence, r(a) is essential and $r(a) \neq R$, Thus, there exists a maximal essential right ideal $\mathrm{M}$ of $\mathrm{R}$ containing $\mathrm{r}(\mathrm{a})$. Hence, $\mathrm{R} / \mathrm{M}$ is simple singular $\mathrm{R}$ - module and so is $\mathrm{N}$ - flat. Since $\mathrm{R} / \mathrm{M}$ is $\mathrm{N}$ - flat, then there exists $d \in M$ and a positive integer $\mathrm{n}$ such that $a^{n} \neq 0$ and $a^{n}=d a^{n}$, since $a^{2}=0$, then $n=1$, so that $a=d a$ and we get $(1-d) \in l(a) \subseteq r(a) \subseteq M$, where $1 \in M$, which is also a contradiction. Hence , $I=0$ and so $\mathrm{R}$ is a semiprime

\section{Definition 3.2 [13]}

The ring $\mathrm{R}$ is called strongly right min-abel if every right minimal idempotent element $e \in R$, is a left semicentral element .

We now consider other condition for right simple singular R-module N-flat to be semiprime.

\section{Theorem 3.3}

Let $\mathrm{R}$ be a strongly right min - abel ring satisfying condition(*). If every simple singular right $\mathrm{R}$ - module is $\mathrm{N}$ - flat, then $\mathrm{R}$ is a semiprime ring.

Proof : Let $a \in R$ satisfyies $a R a=0$. Suppose that $a \neq 0$. Then, there exists a maximal right ideal $\mathrm{M}$ is of $\mathrm{R}$ containing $\mathrm{r}(\mathrm{a})$. If $\mathrm{M}$ is not essential in $\mathrm{R}$. Then , $\mathrm{M}$ is a direct summand of $\mathrm{R}$, since $\mathrm{M}$ is maximal. So we can write $M=r(e)$ for some $0 \neq e^{2}=e \in R, b \in R$ and hence $e b=0$. Because $e R$ is a minimal right ideal of $\mathrm{R}$ and $\mathrm{R}$ is a strongly right min-abel ring, $b e=e b e=0$. Thus , $e \in r(b) \subseteq M=r(e)$, whence $e=0$. This is a contradiction. Therefore, $\mathrm{M}$ must be an essential right ideal of $\mathrm{R}$.Thus , $\mathrm{R} / \mathrm{M}$ is $\mathrm{N}$-flat and so there exists $d \in M$ such that $a=d a$ implies that $(1-d) \in l(a) \subseteq r(a) \subseteq M$ whence $1 \in M$.This is also a contradiction. Hence, $\mathrm{R}$ is a semiprime ring . 


\section{Theorem 3.4}

Let $\mathrm{R}$ be a strongly right min - able with $l\left(a^{n}\right) \subseteq r(a)$ for every $a \in R$ and appositive integer $\mathrm{n}$. If every simple singular right $\mathrm{R}$ - module is $\mathrm{N}$ - flat . Then, $\mathrm{R}$ is a right weakly regular ring .

Proof: We show that $R a R+r(a)=R$ for any $a \in R$. Suppose that there exists $b \in R$ such that $R b R+r(b) \neq R$. Then, there exists a maximal right ideal $\mathrm{M}$ of $\mathrm{R}$ containing $R b R+r(b)$. If $\mathrm{M}$ is not essential in $\mathrm{R}$. Then, $\mathrm{M}$ is a direct summand of $\mathrm{R}$ because $\mathrm{M}$ is maximal .

By a similar method of proof used in Theorem 3.3, $\mathrm{M}$ is an essential right ideal of $\mathrm{R}$. Thus, $\mathrm{R} / \mathrm{M}$ is $\mathrm{N}$ - flat and so there exists a positive integer $\mathrm{n}$ such that $b^{n} \neq 0$ and $b^{n}=c b^{n}$ for some $c \in M$, implies that $(1-c) \in l\left(b^{n}\right) \subseteq r(b) \subseteq M$, whence $1 \in M$, a contradiction. Therefore, $\mathrm{R}$ is a right weakly regular

According to [1], rings satisfying condition (*), and right GQ -injective whose every simple singular right $\mathrm{R}$ - module is $\mathrm{N}$-flat are always regular .But, in general rings satisfying condition (*) and left GQ - injective whose every simple singular right Rmodule is N-flat need not be regular.This leads to the following theorem:

\section{Theorem 3.5}

Let $\mathrm{R}$ be a left GQ - injective, right $\mathrm{MC} 2$ ring whose every simple singular right $\mathrm{R}$ - module is $\mathrm{N}$ - flat and satisfying condition $(*)$. Then, $\mathrm{R}$ is a regular ring .

Proof : First R is left GQ - injective, then $Z(R)=J(R)$ and $R / J(R)$ is a regular ring . From Theorem 2.2, $Z(R)=(0)$. Hence, $J(R)=Z(R)=(0)$, which implies $\mathrm{R}$ is a regular ring

\section{Definition 3.6 [2]}

$\mathrm{R}$ is called a right CAM-ring, if for any maximal essential right ideal $\mathrm{M}$ of $\mathrm{R}$ (if it exits ) and for any right subideal I of $\mathrm{M}$ which is either a complement right subideal of $\mathrm{M}$ or a right annihilator ideal in $\mathrm{R}$, $\mathrm{I}$ is an ideal of $\mathrm{M}$.

Right CAM-ring generalizes semisimple artinian . [2] shows that semiprime right CAM - ring $\mathrm{R}$ is either semisimple artinian or reduced. If $\mathrm{R}$ is also simple singular right $\mathrm{R}$ module is $\mathrm{N}$ - flat, then $\mathrm{R}$ is either semisimple artinian or strongly regular ring. We yield the following theorem :

\section{Theorem 3.7}

Let $\mathrm{R}$ be a wjc right CAM-ring, satisfying condition (*) whose every simple singular right $\mathrm{R}$ - module is $\mathrm{N}$ - flat .Then , $\mathrm{R}$ is either a semisimple artinian or strongly regular ring.

Proof : From Theorem 3.1 $\mathrm{R}$ is semiprime .If $\mathrm{R}$ is not a semisimple artinian, then $\mathrm{R}$ is reduced .Let $0 \neq a \in R$. If $a R \oplus r(a) \neq R$, then $a R \oplus r(a) \subseteq M$ for some maximal right ideal $\mathrm{M}$ of $\mathrm{R}$. If $\mathrm{M}$ is not an essential right ideal of $\mathrm{R}$, then $M=e R$, where $e^{2}=e \in R$. Because $\mathrm{R}$ is reduced, $a e=e a=0$ and $e \in r(a) \subseteq M=r(e)$, a contradiction. Hence, $\mathrm{M}$ is an essential right ideal of $\mathrm{R}$ and so $\mathrm{R} / \mathrm{M}$ is a singular simple right $\mathrm{R}$ - module. Since $\mathrm{R} / \mathrm{M}$ is $\mathrm{N}$ - flat, then there exists $b \in M$ and a positive integer $\mathrm{n}$ such that $a^{n} \neq 0$ and $a^{n}=b a^{n}$. Now, we obtain $(1-b) \in r(a)$, so $1 \in M$, a contradiction . Hence, $a R \oplus r(a)=R$ and then $\mathrm{R}$ is a strongly regular ring . An idempotent $e \in R$ is called right semicentral if $e a=e a e$ for all $a \in R$ [13]. 


\section{Lemma 3.8 [12]}

Let $\mathrm{R}$ be a MERT ring and every right minimal idempotent in $R / J(R)$ is right semicentral .Then, $R / J(R)$ is reduced

\section{Proposition 3.9}

Let $\mathrm{R}$ be a MERT ring and every right minimal idempotent of $R / J(R)$ is right semicentral . If every simple singular right $\mathrm{R}$ - module is $\mathrm{N}$ - flat. Then , $R / J(R)$ is a strongly regular ring.

Proof : By Lemma $3.8, R / J(R)$ is reduced . Let $\overline{0} \neq \bar{a} \in \bar{R}=R / J(R)$. We first that $\bar{R} \bar{a} \bar{R}+r(\bar{a})=\bar{R}$. Suppose that it is not; then there exists a maximal right ideal $\mathrm{M}$ of $\mathrm{R}$ such that $\bar{R} \bar{a} \bar{R}+r(\bar{a}) \subseteq M / J(R)$. Since $\mathrm{R}^{\prime}$ is reduced, $\bar{R} \bar{a} \bar{R}+r(\bar{a})$ is an essential right ideal of $\mathrm{R}^{\prime}$. Therefore , R/M is simple singular right $\mathrm{R}$ - module and so $\mathrm{R} / \mathrm{M}$ is $\mathrm{N}$-flat .

Now, it is easy to show that there exists a positive integer $\mathrm{n}$ and $c \in M$ such that $a^{n} \neq 0$ and $a^{n}=c a^{n}$. Since $\mathrm{M}$ is an ideal of $\mathrm{R}$, we have $1 \in M$, which is a contradiction. Hence, $\bar{R} \bar{a} \bar{R}+r(\bar{a})=\bar{R}$. Let $\bar{a}=\bar{a} \bar{d}$, where $\bar{d} \in \bar{R} \bar{a} \bar{R}$. Then , $(\overline{1}-\bar{d}) \in r(\bar{a})$. If $\bar{a} \bar{R}+r(\bar{a}) \neq \bar{R}$, then there exists a maximal right ideal $\mathrm{L}$ of $\mathrm{R}$ such that $\bar{a} \bar{R}+r(\bar{a}) \subseteq L / J(R)$. Since $R / J(R)$ is reduced, we have $L / J(R)$ is an ideal of $R / J(R)$. Hence, $\mathrm{L}$ is also an ideal of $\mathrm{R}$, and so $\bar{d} \in \bar{R} \bar{a} \bar{R} \subseteq L / J(R)$, and hence $\overline{1} \in L / J(R)$, which is also a contradiction. Thus, $\bar{a} \bar{R}+r(\bar{a})=\bar{R}$, and so $R / J(R)$ is strongly regular ring.

\section{Lemma 3.10 [4]}

For any $a \in \operatorname{Cent}(R)$ ( The Center of $\mathrm{R}$ ), if $a=\operatorname{ara}$ for some $r \in R$; Then there exists $b \in \operatorname{Cent}(R)$ such that $a=a b a$

\section{Lemma 3.11 [4]}

If $\mathrm{R}$ is a semiprime ring ; then $r\left(a^{n}\right)=r(a)$ for any $a \in \operatorname{Cent}(R)$ and a positive integer $n$

\section{Proposition 3.12}

Let $\mathrm{R}$ be a wjc ring satisfying condition (*). If every simple singular right $\mathrm{R}$ module is $\mathrm{N}$ - flat . Then, the Center of $\mathrm{R}$ is a regular ring.

Proof : Let a be a nonzero element in $\operatorname{Cent}(\mathrm{R})$. First, we will show that $a R+r(a)=R$ for any $a \in \operatorname{Cent}(R)$. If not, there exists a maximal right ideal $\mathrm{M}$ of $\mathrm{R}$ such that $a R+r(a) \subseteq M$. Since $a \in \operatorname{Cent}(R), a R+r(a)$ is an essential right ideal and so $\mathrm{M}$ must be an essential right ideal of $\mathrm{R}$. Therefore, $\mathrm{R} / \mathrm{M}$ is $\mathrm{N}$ - flat, so there exists $c \in M$ and a positive integer $\mathrm{n}$ such that $a^{n} \neq 0$ and $a^{n}=c a^{n}=a^{n} c$ implies that $(1-c) \in r\left(a^{n}\right) \subseteq r(a) \subseteq M$ (Theorem 3.1 and Lemma 3.11) and so $1 \in M$, which is a contradiction. Therefore, $a R+r(a)=R$ for any $a \in \operatorname{Cent}(R)$ and so we have $a=$ ara for some $r \in R$. Applying Lemma 3.10, $\operatorname{Cent}(\mathrm{R})$ is a regular ring

\section{Theorem 3.13}

$\mathrm{R}$ is strongly regular ring if and only if $\mathrm{R}$ is a wjc , MERT and 2 - primal ring whose every simple singular right $\mathrm{R}$ - module is $\mathrm{N}$ - flat. 
Proof : First, we show that $\mathrm{R}$ is reduced. In fact, if $a^{2}=0$ for some $0 \neq a \in R$. Then, we have $R a R+r(a)=R$. If not, then there exists a maximal right ideal $\mathrm{M}$ of $\mathrm{R}$ containing $R a R+r(a)$. Observe that $\mathrm{M}$ must be an essential right ideal of $\mathrm{R}$. If not, then $\mathrm{M}$ is a direct summand of $\mathrm{R}$. So we can write $M=r(e)$ for some idempotent e of $\mathrm{R}$. Thus $e R a=0$. Since R is a wjc ring, $a R e=0$ and $a e=0$. Hence, $e \in r(a) \subseteq r(e)$; whence $e=0$, it is a contradiction. Therefore, M must be an essential right ideal of $\mathrm{R}$. Then, $\mathrm{R} / \mathrm{M}$ is $\mathrm{N}$ - flat, there exists a positive integer $\mathrm{n}$ and $b \in M$ such that $a^{n} \neq 0$ and $a^{n}=b a^{n}$. Since $a^{2}=0$, then $n=1$ and therefore $a=b a$ which implies $(1-b) \in M$ and so $1 \in M$ by $\mathrm{M}$ is an ideal of $\mathrm{R}$. This is a contradiction. Hence $R a R+r(a)=R$ and so $a=a d$ for some $d \in R a R$. Since $\mathrm{R}$ is 2 - primal ring, $d \in J(R)$. Hence, $(1-d)$ is right inveritable $\mathrm{v}$ in $\mathrm{R}$ such that $v(1-d)=1$, $v(a-d a)=a$ which yield $a=0$.

Next, we show that $a R+r(a)=R$ for each $a \in R$. If not, then there exists $b \in R$ and a maximal right ideal $\mathrm{L}$ of $\mathrm{R}$ containing $b R+r(b)$. Observe that $\mathrm{L}$ must be essential, so there exists a positive integer $\mathrm{n}$ and $d \in M$ such that $a^{n} \neq 0$ and $a^{n}=d a^{n}$. Now , $(1-d) \in l\left(a^{n}\right)=r\left(a^{n}\right)=r(a) \subseteq L$. so $1 \in L$, which is a contradiction, therefore $a R+r(a)=R$. Hence, $\mathrm{R}$ is strongly regular ring .

Conversely : it is obvious. 


\section{$\underline{\text { REFERENCES }}$}

[1] Mahmood, R.D. and Husam Q.M. (2010) ; "On N-flat rings", Received, J. Al Rafiden of Comp. and Math.

[2] Ming , R.Y.C. (1983) ; " On Quasi-Frobeniusean and artinian Rings", Pub. De Linstitute Mathématique, 33(47), pp. 239-245.

[3] Ming , R.Y.C. (1995); " On Von Neumann, regularity, injectivity and flatness", Yokohama Math., Vol. 43, pp.37-44 .

[4] Nam, S.B. (1999) ; "A note on simple singular GP-injective modules", J. Kangweon - Kyungki. Mth. J. 7(2), pp.215-218.

[5] Nicholson, W.K. and Yousif , M. F. (1997) ;" Mininjevtive rings " , J. Algebra, Vol. 187 , pp. 548-578.

[6] Nicholson , W.K. and Yousif M.F. (2004) ; "Weakly continuous and C2 - rings" , Comm. In Algebra, pp. 2429-2466.

[7] Ramamurthi, V.S. (1975); "On injectivity and flatness of certain cyclic modules" , Proc. Amer. Math. Soc., 48, pp.21- 25.

[8] Rege , M.B. (1986) ; " On Von Neumann regular rings and SF- rings" , Math. Japonica , 31(6), pp. 927-936.

[9] Von Neumann J. (1936); " On regular rings " , Proc. Nat. Sci. U.S.A. 22, pp. 707-713.

[10] Wei, J.C. (2005) ; " The rings characterized by minimal left ideal" , Acta. Math. Sinica, English Series 21(3), pp. 473- 482.

[11] Wei , J.C. and Chen, J.H. (2007) ; " Nil-injective rings " , Inter . J. of Algebra 2, pp. 1-21.

[12] Wei , J.C. (2007); "On simple singular YJ- injective modules", Sou. Asiam Bull. Of Math. 31, pp. 1 - 10.

[13] Wei , J.C. (2008); "Certain rings whose simple singular modules are niliniective", Turk J. Math .32, pp. 393 - 408. 